Article

\title{
Long-Term Incubation PrPCWD with Soils Affects Prion Recovery but Not Infectivity
}

\author{
Alsu Kuznetsova ${ }^{1} \mathbb{D}$, Debbie McKenzie ${ }^{2}$, Catherine Cullingham ${ }^{3} \mathbb{D}$ and Judd M. Aiken ${ }^{1, *}$ \\ 1 Agricultural, Life and Environmental Sciences Faculty, University of Alberta, Edmonton, AB T6G 2G8, \\ Canada; alsu@ualberta.ca \\ 2 Faculty of Science, University of Alberta, Edmonton, AB T6G 2M8, Canada; debbie.mckenzie@ualberta.ca \\ 3 Department of Biology, Carleton University, Ottawa, ON K1S 5B6, Canada; catherine.cullingham@carleton.ca \\ * Correspondence: judd.aiken@ualberta.ca
}

Received: 10 March 2020; Accepted: 21 April 2020; Published: 23 April 2020

\begin{abstract}
Chronic wasting disease (CWD) is a contagious prion disease of cervids. The infectious agent is shed from animals at the preclinical and clinical stages of disease where it persists in the environment as a reservoir of CWD infectivity. In this study, we demonstrate that long-term incubation of CWD prions (generated from tg-mice infected with deer or elk prions) with illite, montmorillonite (Mte) and whole soils results in decreased recovery of $\mathrm{PrP}^{\mathrm{CWD}}$, suggesting that binding becomes more avid and irreversible with time. This continual decline of immunoblot $\operatorname{PrPCWD}$ detection did not correlate with prion infectivity levels. Bioassay showed no significant differences in incubation periods between mice inoculated with $1 \%$ CWD brain homogenate $(\mathrm{BH})$ and with the CWD-BH pre-incubated with quartz or Luvisolic Ae horizon for 1 or 30 weeks. After 55 weeks incubation with Chernozem and Luvisol, bound $\mathrm{PrP}^{\mathrm{CWD}}$ was not detectable by immunoblotting but remained infectious. This study shows that although recovery of $\mathrm{PrP}^{\mathrm{CWD}}$ bound to soil minerals and whole soils with time become more difficult, prion infectivity is not significantly altered. Detection of prions in soil is, therefore, not only affected by soil type but also by length of time of the prion-soil interaction.
\end{abstract}

Keywords: prion protein; soil; CWD; prolonged incubation; CWD infectivity; prion detection

\section{Introduction}

Chronic wasting disease (CWD), a contagious prion disease affecting different cervid species, has been present in the United States for at least 50 years. Initially identified in the western U.S., the disease has spread through much of western and central North America. The disease has also been confirmed in Korean and European cervids [1]. A significant challenge for disease management and surveillance is that infectious CWD prions are shed during both the preclinical and clinical stages of disease [2-4]. The shed prions can remain infectious even after prolonged periods in the environment [5-7]. Upper soil horizons can serve as an environmental reservoir for prions [8-12], and, therefore, facilitate horizontal transmission of CWD [13,14].

Soil properties are a key factor for CWD persistence and transmission in the environment $[8,15,16]$. Whereas the soil mineral montmorillonite (Mte) avidly binds abnormal prion protein, enhancing infectivity [17,18], an organic soil constituent, humic acids can decrease CWD infectivity [19]. Prion persistence under harsh environmental conditions has been described [20,21], demonstrating prions shed in native soil maintain infectivity for years [6,22]. In addition, laboratory soil column experiments indicated that $\mathrm{PrP}^{\mathrm{Sc}}$ was stable after incubation in the soil for 18 months [23], and soil-bound hamster prions provided a catalytically active seed for protein misfolding cyclic amplification (PMCA) after a year-long incubation [24]. These data suggest that soil is likely to be an important environmental reservoir of prion infectivity. One study demonstrated the presence of $\mathrm{PrP}^{\mathrm{Sc}}$ in naturally contaminated 
soils [12]. A number of laboratory studies have, however, suggested a time-dependent decline in the ability to detect rodent prions when bound to soils [22,25]. A decline in recoverable $\mathrm{PrP}^{\mathrm{PC}}$, after prolonged incubation, was found to be soil type specific with less $\mathrm{PrP}^{\mathrm{Sc}}$ recovery from clay compared to sandy soils [23].

Sensitive detection of infectious CWD prions from environmental samples would have clear benefits for monitoring CWD spread. We demonstrate that, with time, $\operatorname{PrP}^{\mathrm{CWD}}$ becomes more difficult to recover from variety of soil types, yet CWD infectivity does not significantly decrease. Our findings suggest significant limitations to soil-bound $\mathrm{PrP}^{\mathrm{CWD}}$ detection especially for soils with montmorillonite minerology, impacting both surveillance and mitigation approaches.

\section{Materials and Methods}

\subsection{Soils and Minerals}

We studied eight surface soil horizons from 7 different soils: horizons LFH (plant litter horizon) and Ae (illite-enriched mineral horizon) of Luvisol, horizons LFH (plant litter) and Bf (illite- and iron-enriched mineral horizon) of Brunisol, Ah (humic) horizons of two Chernozemic soils, and Ae (quartz-illite mineral horizon) of two Podzolic soils. All soil samples (except Podzols) were collected in Canada and represent soil cover of boreal, tundra and prairie regions. Podzols, Luvisols and Brunisols are found in boreal and tundra ecozones, while the Chernozems are dominant in the prairies [8]. Properties of the soils are summarized in Kuznetsova et al. [24]. Minerals used for binding experiments were purchased from Ward's Science: quartz (\#940005), illite (\#46E0315) and montmorillonite (Mte, \#46E0435).

\subsection{Ethics Statement}

All work with animals was performed in compliance with the Canadian Council on Animal Care Guidelines and Policies. All procedures involving animals were reviewed and approved by the Health Sciences Animal Care and Use Committee of the University of Alberta.

\subsection{Prion Preparation, Binding Experiments and Immunoblotting}

CWD-infected brain homogenates were prepared from transgenic mice expressing either 132MM elk PrP (tgElk) or wild-type white-tailed deer PrP (tg33) infected with elk or white-tailed deer prions. Uninfected brain homogenates were prepared from non-infected tgElk or tg33 mice. Brain tissues were homogenized $(10 \% w / v)$ in water and, for some experiments, clarified at $800 \mathrm{~g}$ for $5 \mathrm{~min}$ [11]. In binding experiments, identical amounts of $10 \%$ brain homogenate $\left(\mathrm{BH}^{\mathrm{CWD}}\right.$ or uninfected $\mathrm{NBH}$; final concentration $0.25 \%$ ) were incubated with water (control) or a binding agent (solid phase minerals or soil horizons) in silanized Eppendorf tubes at $4{ }^{\circ} \mathrm{C}$. To minimize non-specific binding of PrP to the plastic tube walls, the tubes were treated with chlorodimethylsilane (silanized tubes) before the experiment. Samples were collected at specific time points; proteins were extracted with $40 \mu \mathrm{L}$ of a 5xSDS sample buffer at $100{ }^{\circ} \mathrm{C}$ for $10 \mathrm{~min}$, and stored at $-80^{\circ} \mathrm{C}$ until analyzed by immunoblotting. These samples are not protease digested as the PK binds to the soils and the extraction conditions, SDS and boiling, resulting in denatured PK-sensitive prion protein. Samples $(10 \mu \mathrm{L})$ were resolved on 12or 15-well 12\% NuPAGE bis-Tris gels (Invitrogen), transferred to PVDF membrane and probed with the anti-PrP antibody Bar 224 for PrPCWD .

\subsection{Animal Bioassay}

tgElk mice were inoculated intraperitoneally (i.p.) with $100 \mu \mathrm{L}$ of $1 \% \mathrm{BH}$ (from transgenic mice infected with elk prions) collected after 1 and 30 weeks incubation with quartz and Luvisolic Ae horizon. Soil/mineral samples were re-suspended in water, and pasteurized $10 \mathrm{~min}$ at $80^{\circ} \mathrm{C}$. Controls included the inoculation of equivalent amounts of $1 \%$ CWD-BH or uninfected $\mathrm{BH}(1 \%)$ incubated for 1 week at $4{ }^{\circ} \mathrm{C}$. In a separate study, $\mathrm{BH}(1 \%$ tgElk-CWD) were incubated with Luvisolic LFH and Ae and 
Chernozemic Ah horizons for 1 and 55 weeks, the samples were re-suspended in water, pasteurized $10 \mathrm{~min}$ at $80^{\circ} \mathrm{C}$, and $100 \mu \mathrm{L}$ were inoculated (i.p.) into tgElk mice. CWD-BH (1\% and $\left.0.01 \%\right)$ incubated for 1 week or uninfected $\mathrm{BH}(1 \%)$ were also pasteurized and inoculated as controls. In both bioassays, mice were monitored daily for the onset of clinical signs and euthanized upon confirmed clinical disease. Brains from clinically positive mice and uninfected controls were analyzed for protease-resistant $\operatorname{PrP}\left(\mathrm{PrP}^{\mathrm{res}}\right)$ by immunoblotting as described above. For the proteinase digestion reactions, $50 \mu \mathrm{g}$ total proteins were treated with $75 \mu \mathrm{g} / \mathrm{mL}$ of proteinase $\mathrm{K}$ (Life Technologies) for $45 \mathrm{~min}$ at $37^{\circ} \mathrm{C}$. Reactions were terminated by adding of the $40 \mu \mathrm{L}$ of $5 x \operatorname{SDS}$ sample buffer and boiling at $100{ }^{\circ} \mathrm{C}$ for $10 \mathrm{~min}$.

\section{Results and Discussion}

\subsection{Decline in PrPCWD Recovery with Increasing Soil/Mineral Incubation Time}

Long-term incubation of CWD-infected $\mathrm{BH}$ with illite, Mte and soil horizons results in a time-dependent decline in recovery of $\mathrm{PrPCWD}^{\mathrm{C}}$ (Figure 1). After one week of incubation, $\operatorname{PrPCWD}$ signal intensity decreased for all mineral/soil combinations, except quartz, in comparison to the control (1\% BH diluted in water without solid substrate) (Figure 1A). Samples were collected at specified time points over 7 weeks and were treated with SDS (diluted to a final concentration $0.14 \%$ of $\mathrm{BH}$ ) and incubated at $100{ }^{\circ} \mathrm{C}$, a procedure from which we have successfully recovered prions avidly bound to clay minerals [11]. After 7 weeks of incubation, PrPCWD was not detectable, by immunoblot, from illite, Mte, Brunisolic LFH and both Chernozemic horizon samples, but still detected in Luvisolic horizons (plant litter LFH and eluvial Ae horizon), and weakly detected for Bf Brunisolic horizon. PrPCWD was detectable in both the $\mathrm{BH}+$ quartz and $\mathrm{BH}$ control throughout the 7 weeks (Figure 1). Although the PrP signal after incubation with quartz did not decline, we observed the glycosylation pattern became smeared (Figure 1A-D). For all other minerals and soil horizons PrP desorption is characterized by truncation of the N-terminal fragment $(\sim 35 \mathrm{kDa})$; this cleavage has been described previously for Mte and the fate of the cleaved amino acid residues has not been determined [11,23]. Similar experiments with uninfected brain homogenate resulted in no detection of $\operatorname{PrP}^{\mathrm{C}}$ after 10 days of incubation with soils or soil minerals (Figure 1E) suggesting that the PrP detected following extended soil/soil mineral incubations is PrPCWD.

We repeated the experiment for longer periods of time (up to 55 weeks) using the same minerals and soils (Qz, illite, Mte, Luvisol, Brunisol and Chernozems) and found a similar trend. After prolonged incubation of 15 weeks, PrPCWD signal from $\mathrm{BH}$ or $\mathrm{BH}+$ quartz signal was stable (Figure 2A), while incubation with illite resulted in reduced PrPCWD signal. After 15 weeks incubation with two Luvisolic horizons (LFH and Ae), PrP detection continued to decrease (Figure 2B) in comparison to the BH control (last band, Figure 2B). Comparison between the samples after 20 weeks of incubation revealed that PrPCWD signal in $\mathrm{BH}$ control or $\mathrm{BH}+$ quartz signal were similarly intense (Figure $2 \mathrm{C}$ ), but $\mathrm{PrPCWD}^{\mathrm{CW}}$ was not detectable from the illite and Mte samples. Amongst the soils, the strongest PrPCWD detection was from the Luvisolic horizons, followed by the Brunisols, with the lowest PrPCWD recovery associated with the Chernozems. After 30 weeks incubation, PrPCWD signal from BH remained the same, but was no longer detectable in any soil from this set (Figure 2D). Incubation of CWD-BH with 2 Podzol soils, sandy soils with low humus content collected in Scandinavia, showed stable PrP signal during the first 28 weeks (Figure 2E) while exhibiting a decline after 100 weeks of incubation (Figure 2F). Across the soils and time-points, recovery of PrPCWD was higher from the Podzolic and Luvisolic soils, followed by the Brunisols, and finally the Chernozems. Variation in soil types and differences in their properties such as minerology, clay and humus content, are likely the main factors responsible for PrP persistence and recovery after prolonged incubation. 

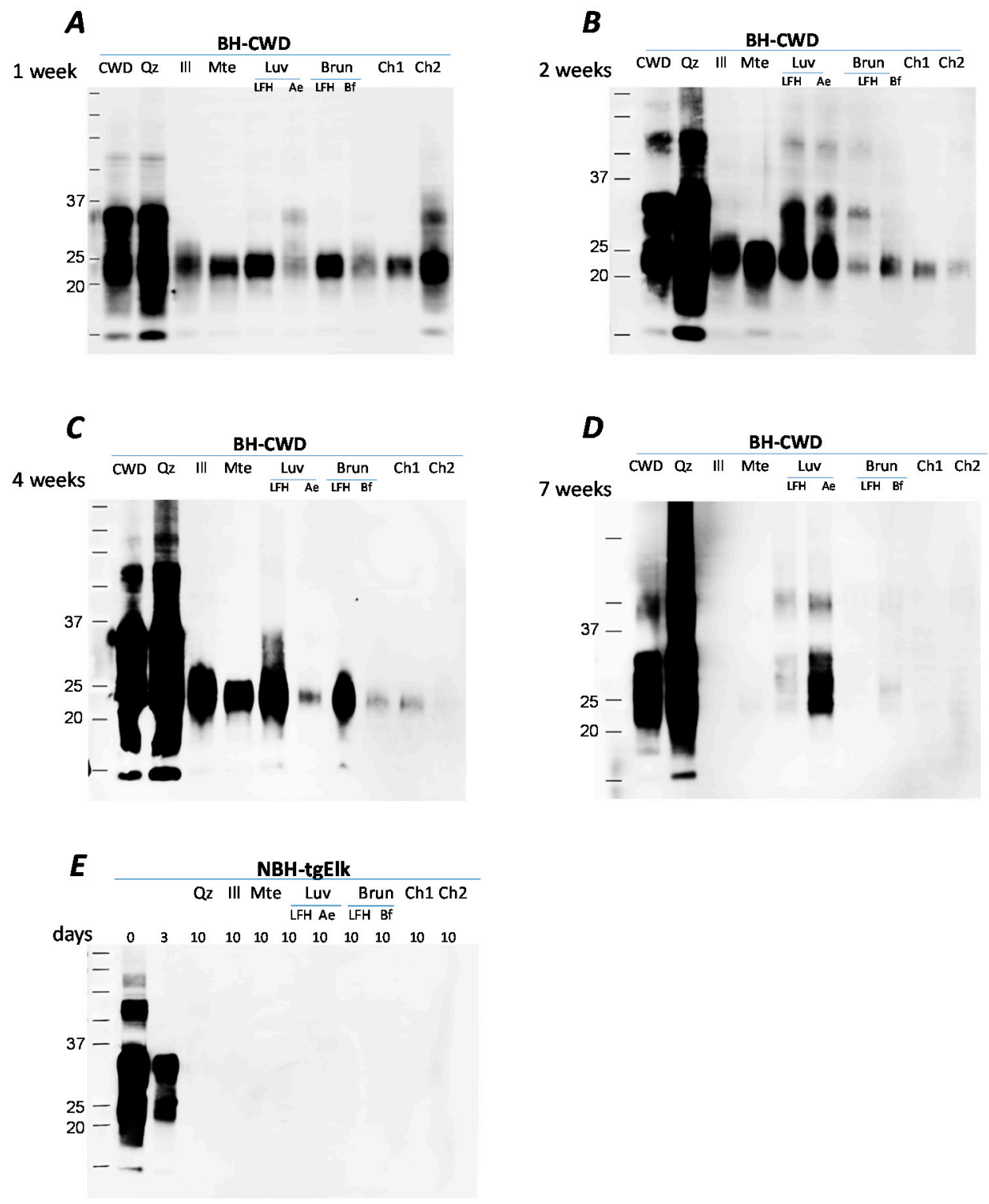

Figure 1. Decreasing PrP signal after incubation with soils and soil minerals for 7 weeks. Proteins were extracted after 1 (Panel A), 2 (Panel B), 4 (Panel C) or 7 (Panel D) weeks incubation with water (control), quartz, illite, Mte, Luvisolic horizons LFH or Ae, Brunisolic horizons LFH and Bf and Ah horizons from 2 Chernozems. (Panel E): uninfected brain homogenate (BH) incubated for 0, 3 and 10 days. Identical amounts of $1 \%$ chronic wasting disease (CWD)-infected or uninfected BH (tgElk-CWD, final concentration $0.25 \%$ ) were incubated with water (control) soils or soil minerals at $4{ }^{\circ} \mathrm{C}$ in silanized tubes. Samples were taken at specified time points and analyzed by western blot using Bar224 antibody. 
$\boldsymbol{A}$

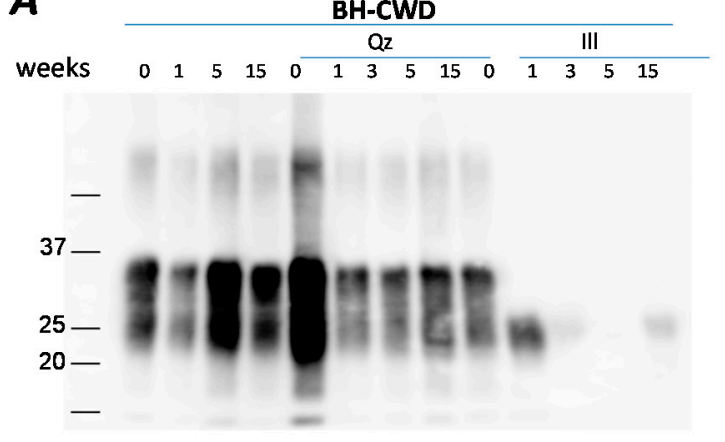

B

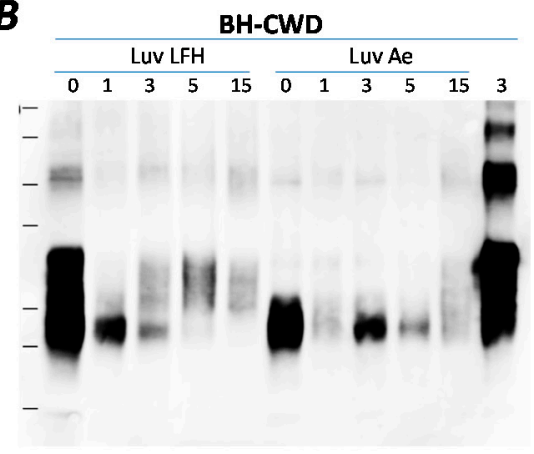

BH-CWD

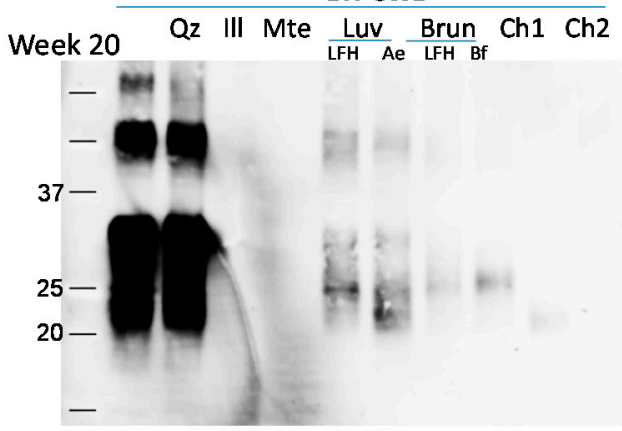

$\boldsymbol{D}$
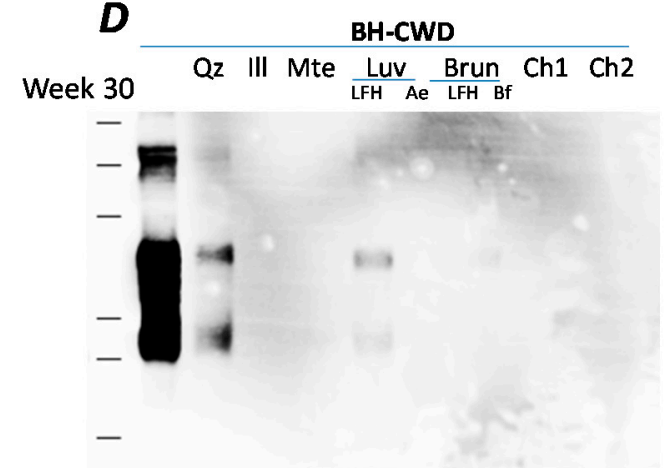

$\boldsymbol{F}$
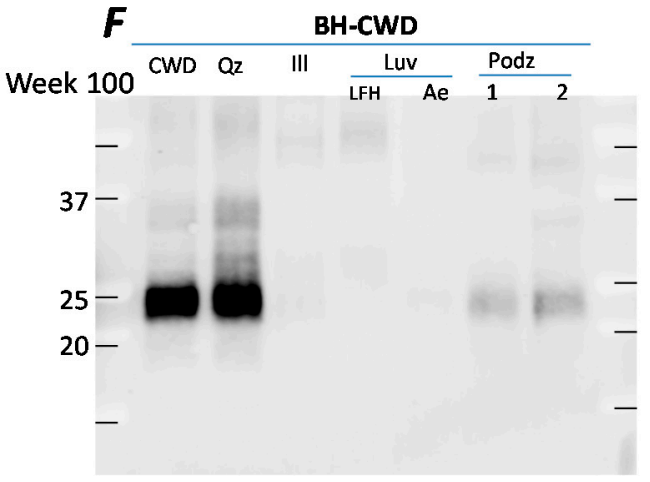

Figure 2. PrP extracted after incubation with soils or soil minerals. (A) $\operatorname{PrP}$ after 1, 5 or 15 weeks incubation with water (control), quartz or illite; (B) PrP after 1, 5 or 15 weeks incubation with Luvisolic horizons LFH or Ae; (C) PrP after 20 weeks incubation with quartz, illite, Mte, Luvisolic horizons LFH or Ae, Brunisolic horizons LFH and Bf and Ah horizons from 2 Chernozems; (D) PrP signal extracted after 30 weeks incubation; (E,F) PrP after incubation with soils and soil minerals for 28 and 100 weeks incubation with water (control), quartz, illite, Mte, Luvisolic horizons LFH and Ae, and Ae horizons from two Podzols. Identical amounts of 1\% CWD-infected brain homogenate from tg 33 mice ((Panels A-D); final concentration 0.25\%) and tgElk mice ((Panels E,F); final concentration 0.25\%) were incubated with water (control) or $15 \mathrm{mg} / \mathrm{mL}$ suspension of soils/soil minerals at $4{ }^{\circ} \mathrm{C}$ in silanized tubes. Samples were taken at specific time points and analyzed by western blot using Bar224 antibody.

\subsection{Mineral/Soil Interaction Has Little Effect on Infectivity}

Animal bioassay experiments of Luvisolic Ae horizon (1, 30 and 55 week incubation) and Chernozemic Ah horizon ( 1 and 55 week incubation) were performed to determine the effect of prolonged soil mineral/soil interaction on infectivity (Figure 3). Infectious brain homogenate (no soil mineral) and quartz sand (little decline in $\mathrm{PrP}^{\mathrm{CWD}}$ with prolonged incubation) were included as controls. Extended incubation of CWD infectivity with soil horizons had little effect on infectivity. 
Thirty week and 55 week incubation of Luvisolic Ae horizon produced similar infectivity to 1 week Luvisolic Ae. Similarly, Chernozemic Ah horizon bound to CWD for 55 weeks resulted in infectivity levels similar to the 1 week soil/PrPCWD interaction (Figure 3A).
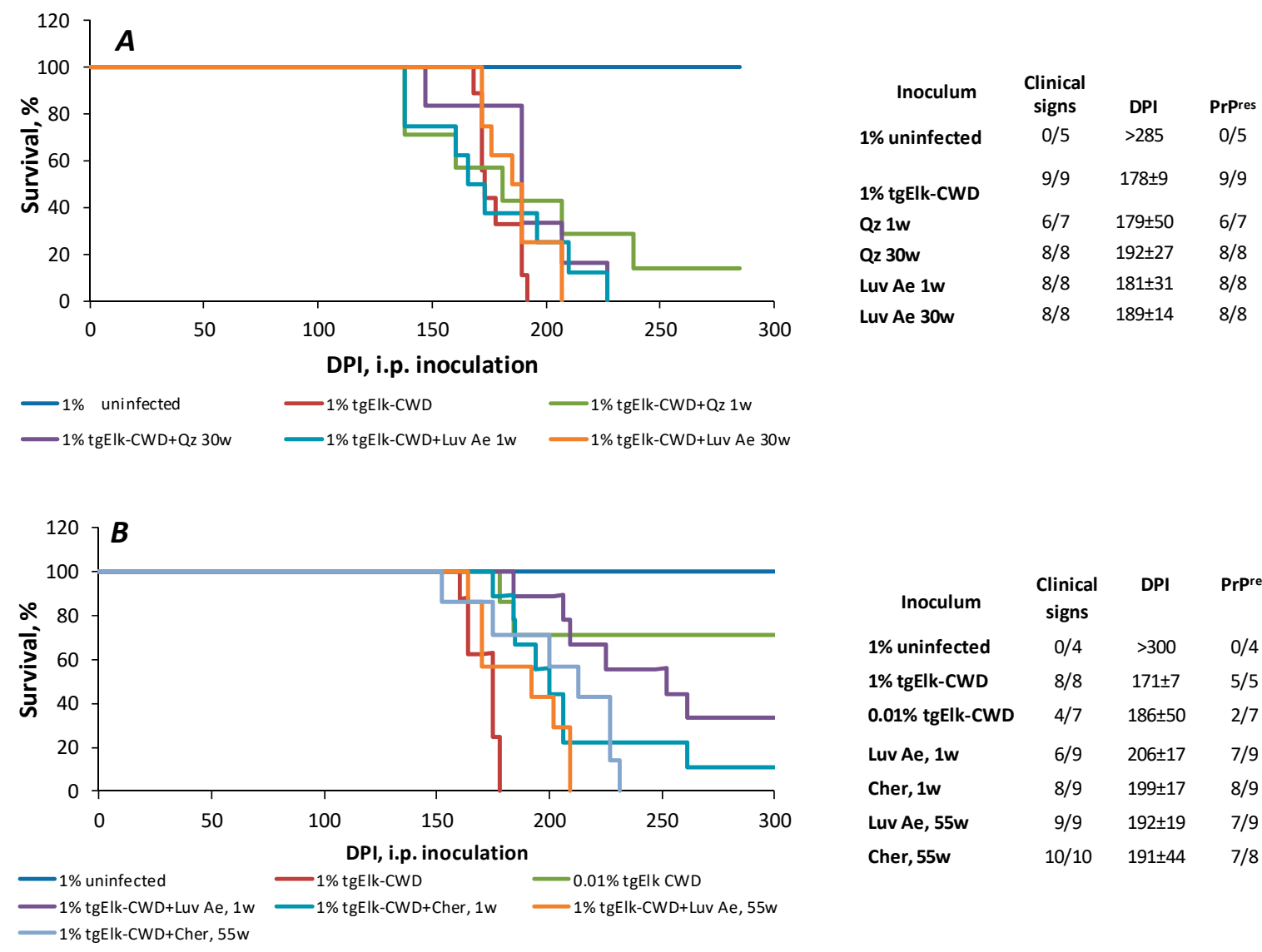

\begin{tabular}{lccc}
\multicolumn{1}{c}{ Inoculum } & $\begin{array}{c}\text { Clinical } \\
\text { signs }\end{array}$ & DPI & PrPres \\
1\% uninfected & $0 / 4$ & $>300$ & $0 / 4$ \\
1\% tgElk-CWD & $8 / 8$ & $171 \pm 7$ & $5 / 5$ \\
0.01\% tgElk-CWD & $4 / 7$ & $186 \pm 50$ & $2 / 7$ \\
Luv Ae, 1w & $6 / 9$ & $206 \pm 17$ & $7 / 9$ \\
Cher, 1w & $8 / 9$ & $199 \pm 17$ & $8 / 9$ \\
Luv Ae, 55w & $9 / 9$ & $192 \pm 19$ & $7 / 9$ \\
Cher, 55w & $10 / 10$ & $191 \pm 44$ & $7 / 8$
\end{tabular}

Figure 3. Animal bioassays. (A) Survival curve of tgElk mice i.p. inoculated with 1\% CWD-BH incubated with quartz (Qz) and Luvisolic Ae horizon for 1 and 30 weeks; (B) Survival curve of tgElk mice i.p. inoculated with 1\% CWD-BH incubated with Luvisolic Ae and Chernozemic Ah horizons for 1 week and 55 weeks.

Similar to the previous experiments, the amount of detectable PrP recovered from the inoculums for this bioassay differed with $\mathrm{PrP}$ being detectable for 1\% and $0.01 \% \mathrm{CWD}-\mathrm{BH}$ control as well as for 1 week old $\mathrm{BH}+$ Luvisolic Ae horizon, while the CWD-BH/soil samples incubated 55 weeks were not detectable by immunoblotting (Supplementary Figure S2). All control animals inoculated with $1 \% \mathrm{BH}$ (unbound PrP, control) exhibited clinical signs by $171 \pm 7 \mathrm{dpi}$ (Figure 3B). While four out of seven mice inoculated with $0.01 \% \mathrm{BH}$ (elk-CWD) showed clinical signs (186 $\pm 50 \mathrm{dpi}$ ), most mice inoculated with soil samples (CWD-BH pre-incubated with soils) exhibited clinical signs and PrPres accumulation in brain. There is no significant difference between inocula in incubation periods; mice inoculated with soil samples have an incubation period of appx. $200 \mathrm{dpi}$ with a wide standard deviation. Exact incubation periods for each inoculum and PrP accumulation in harvested brains are shown in Figure 3, Supplementary Figures S3 and S4, respectively.

\subsection{Declining Prion Recovery Yet Infectivity Remains}

To summarize, the difference between inocula, as detected by immunoblotting (no signal after prolonged incubation with soils) did not correlate with the incubation period. The bioassay did not suggest a decrease in infectivity of soil-bound prions or PrP degradation. There are several possible explanations for the observed decline in immunoblot $\mathrm{PrP}^{\mathrm{CWD}}$ signal upon prolonged incubation with 
soils and minerals, including adsorption to soil, adsorption to the tube, or inactivation of the antibody epitope. To minimize adsorption to Eppendorf tubes, we coated them with chlorodimethylsilane. Note that, the $\mathrm{BH}$ alone serves as a sorption control; if adsorption was a significant factor, we would observe decreasing levels of $\mathrm{PrP}^{\mathrm{CWD}}$ for the $\mathrm{BH}$ control, however it was stable over 30 weeks (Figure 2). To determine whether the antibody epitope was removed we used mAb D15.15 (epitope 176-178), SAF83 (epitope 126-164 excl. 142-160) and 8G8 (epitope 97-102), in addition to the mAb Bar224 (epitope 141-151). We obtained similar results for all antibodies where PrP signal remained stable for the BH control sample and decline or disappeared in samples with soils (Supplementary Figure S1). The data suggest the most probable explanation that the $\mathrm{PrP}^{\mathrm{CWD}}$ adsorption to soil might increase with time because it depends on competitive sorption effects of the brain homogenate matrix where $\mathrm{PrPCWD}$ initially competes for sorption sites with thousands of other constituents. Over time, more surface area in soils may become available due to degradation, reorientation, or conformational changes of bound molecules, allowing more stable PrPCWD to adsorb. We previously demonstrated that prion binding to some soils enhances infectivity [17]. It is, therefore, possible that $\operatorname{PrP}^{\mathrm{CWD}}$ levels declined with extended soil incubations but infectivity levels are enhanced. This possibility does not alter our conclusion that declining ability to detect $\mathrm{PrP}^{\mathrm{CWD}}$ when bound to soils for extended times does not result in a concomitant decline in CWD infectivity.

Clearly, the binding of whole soils and soil minerals is complex and the interaction can be exceedingly avid. Even short incubation times with the soil mineral montmorillonite require extreme conditions $\left(10 \%\right.$ SDS, $\left.100{ }^{\circ} \mathrm{C}\right)$ to facilitate recovery of $\mathrm{PrP}^{\mathrm{CWD}}$. The decline in $\operatorname{PrPCWD}$ recovery with increasing incubation time varied with soil type. Soils from prairie regions (montmorillonite mineralogy and high humus content) show the highest ability to bind $\operatorname{PrPCWD}$, where $\operatorname{Pr} \mathrm{P}^{\mathrm{CWD}}$ was not recoverable after 4 weeks of incubation with those soils. Prion incubation with soils from boreal regions (illite/quartz mineralogy, low humus content) result in more stable recovery of PrPCWD signal, recoverable up to 28 weeks from Podzols and up to 20 weeks from Luvisols and Brunisols.

Sensitive detection of $\mathrm{PrP}^{\mathrm{CWD}}$ prions from environmental samples would greatly simplify disease monitoring. The declining ability with time to detect CWD prions bound to soils significantly complicates such an approach. Alternative methods of extractions of prions from soil are required, and extractions that retain $\mathrm{PrP}^{\mathrm{CWD}}$ structure would facilitate sensitive prion amplification technologies (e.g., PMCA, RT-QuIC). The ability to in vitro amplify soil-bound prions varies significantly with soil type; it is significantly reduced when bound to clay soil and soil organic matter compared to unbound and sand-bound $\mathrm{PrP}^{\mathrm{Sc}}$ [26]. Although in vitro amplification technologies are the most sensitive and promising method to detect soil-bound prions, and were used to detect $\operatorname{PrP}^{\mathrm{CWD}}$ in naturally contaminated water and soil samples [12], $\mathrm{PrP}^{\mathrm{CWD}}$ recovery efficiency is not sufficient to detect infectious prions in a wide range of environmental samples.

\section{Conclusions}

The binding of prions to soil minerals and other soil constituents impacts $\operatorname{PrP}^{\mathrm{CWD}}$ recovery. During extended incubation with soils, PrP signal on immunoblots continuously declined until it was no longer detectable after 25 weeks in soils with loamy-clay texture and Mte minerology. $\mathrm{PrP}^{\mathrm{CWD}}$ infectivity did not, however, decrease after 30 weeks incubation with quartz and the Luvisolic Ae soil horizon. At 55 weeks incubation in Chernozem and Luvisol, CWD-BH remained infectious. We studied a wide variety of soil types (from prairie, mountain and boreal regions) and showed decreased $\operatorname{Pr} \mathrm{P}^{\mathrm{CWD}}$ signal recovery (as measured by immunoblotting) with retention of infectivity. The decrease in $\mathrm{PrP}^{\mathrm{CWD}}$ recovery was particularly dramatic in soils from the prairie region. Regardless of soil minerology, texture and humus content, detection of $\mathrm{PrP}^{\mathrm{CWD}}$ in environmental soil samples is a challenge after long-term incubation. These findings provide important information on the behavior of prions in natural environments, but complicate analysis of environmental samples. 
Supplementary Materials: The following are available online at http://www.mdpi.com/2076-0817/9/4/311/s1, Figure S1: Decreasing of PrP signal extracted after incubation with soils and soil minerals for 5 weeks; Figure S2: Inoculum for animal bioassay with tgElk mice i.p. inoculated with 1\% CWD-BH incubated with Luvisolic Ae, LFH and Chernozemic Ah horizons for 1 and 55 weeks; Figure S3: Accumulation of PrPres in brains harvested from mice with clinical signs. Numbers represent days after inoculation when animal was harvested; Figure S4: Accumulation of $\mathrm{PrP}^{\mathrm{res}}$ in brains harvested from mice with clinical signal and at the end of experiment (without clinical signals).

Author Contributions: Conceptualization and drafting the manuscript: A.K., J.M.A. and D.M.; Data analysis: A.K., J.M.A.; Sample collection and processing: A.K.; Writing-review and editing: all authors, A.K., D.M., C.C., and J.M.A.; project administration: C.C. and D.M.; funding acquisition: J.M.A. and D.M. All authors have read and agreed to the published version of the manuscript.

Funding: This work was supported by Alberta Innovates BioSolutions through the Alberta Prion Research Institute grants 200900188, 201300025 and PAA13007, and by Genome Canada, the University of Alberta, and the Alberta Prion Research Institute and Alberta Agriculture and Forestry, through Genome Alberta in support of the Systems Biology and Molecular Ecology of Chronic Wasting Disease project.

Conflicts of Interest: The authors declare no conflict of interest. The funders had no role in the design of the study; in the collection, analyses, or interpretation of data; in the writing of the manuscript, or in the decision to publish the results.

\section{References}

1. Benestad, S.L.; Mitchell, G.; Simmons, M.; Ytrehus, B.; Vikøren, T. First case of chronic wasting disease in Europe in a Norwegian free-ranging reindeer. Vet. Res. 2016, 47, 1. [CrossRef] [PubMed]

2. Henderson, D.M.; Denkers, N.D.; Hoover, C.E.; Garbino, N.; Mathiason, C.K.; Hoover, E.A. Longitudinal detection of prion shedding in saliva and urine by chronic wasting disease-infected deer by real-time quaking-induced conversion. J. Virol. 2015, 89, 9338-9347. [CrossRef] [PubMed]

3. Haley, N.J.; Mathiason, C.K.; Carver, S.; Zabel, M.; Telling, G.C.; Hoover, E.A. Detection of chronic wasting disease prions in salivary, urinary, and intestinal tissues of deer: Potential mechanisms of prion shedding and transmission. J. Virol. 2011, 85, 6309-6318. [CrossRef] [PubMed]

4. Tamgüney, G.; Miller, M.W.; Wolfe, L.L.; Sirochman, T.M.; Glidden, D.V.; Palmer, C.; Lemus, A.; Dearmond, S.J.; Prusiner, S.B. Asymptomatic deer excrete infectious prions in faeces. Nature 2009, 461, 529-532. [CrossRef] [PubMed]

5. Brown, P.; Gajdusek, D.C. Survival of scrapie virus after 3 years' interment. Lancet 1991, 337, $269-270$. [CrossRef]

6. Georgsson, G.; Sigurdarson, S.; Brown, P. Infectious agent of sheep scrapie may persist in the environment for at least 16 years. J. Gen. Virol. 2006, 87, 3737-3740. [CrossRef] [PubMed]

7. Pritzkow, S.; Morales, R.; Lyon, A.; Concha-Marambio, L.; Urayama, A.; Soto, C. Efficient prion disease transmission through common environmental materials. J. Biol. Chem. 2018, 293, 3363-3373. [CrossRef]

8. Kuznetsova, A.; McKenzie, D.; Banser, P.; Siddique, T.; Aiken, J.M. Potential role of soil properties in the spread of CWD in western Canada. Prion 2014, 8, 92-99. [CrossRef]

9. Saunders, S.E.; Bartz, J.C.; Bartelt-Hunt, S.L. Soil-mediated prion transmission: Is local soil-type a key determinant of prion disease incidence? Chemosphere 2012, 87, 661-667. [CrossRef]

10. Smith, C.B.; Booth, C.J.; Pedersen, J.A. Fate of prions in soil: A review. J. Environ. Qual. 2011, 40, 449-461. [CrossRef]

11. Johnson, C.J.; Phillips, K.E.; Schramm, P.T.; McKenzie, D.; Aiken, J.M.; Pedersen, J.A. Prions adhere to soil minerals and remain infectious. PLoS Pathog. 2006, 2, 296-302. [CrossRef] [PubMed]

12. Plummer, I.H.; Johnson, C.J.; Chesney, A.R.; Pedersen, J.A.; Samuel, M.D. Mineral licks as environmental reservoirs of chronic wasting disease prions. PLoS ONE 2018, 13, e0196745. [CrossRef] [PubMed]

13. Gough, K.C.; Maddison, B.C. Prion transmission: Prion excretion and occurrence in the environment. Prion 2010, 4, 275-282. [CrossRef] [PubMed]

14. Mathiason, C.K.; Powers, J.G.; Dahmes, S.J.; Osborn, D.A.; Miller, K.V.; Warren, R.J.; Mason, G.L.; Hays, S.A.; Hayes-Klug, J.; Seelig, D.M.; et al. Infectious prions in the saliva and blood of deer with chronic wasting disease. Science 2006, 314, 133-136. [CrossRef] [PubMed] 
15. Dorak, S.J.; Green, M.L.; Wander, M.M.; Ruiz, M.O.; Buhnerkempe, M.G.; Tian, T.; Novakofski, J.E.; Mateus-Pinilla, N.E. Clay content and pH: Soil characteristic associations with the persistent presence of chronic wasting disease in northern Illinois. Sci. Rep. 2017, 7. [CrossRef] [PubMed]

16. Walter, D.W.; Walsh, D.P.; Farnsworth, M.L.; Winkelman, D.L.; Miller, M.W. Soil clay content underlies prion infection odds. Nat. Commun. 2011, 2. [CrossRef]

17. Johnson, C.J.; Pedersen, J.A.; Chappell, R.J.; McKenzie, D.; Aiken, J.M. Oral transmissibility of prion disease is enhanced by binding to soil particles. PLoS Pathog. 2007, 3, e93. [CrossRef]

18. Wyckoff, A.C.; Kane, S.; Lockwood, K.; Seligman, J.; Michel, B.; Hill, D.; Ortega, A.; Mangalea, M.R.; Telling, G.C.; Miller, M.W.; et al. Clay components in soil dictate environmental stability and bioavailability of cervid prions in mice. Front. Microbiol. 2016, 7, 1885. [CrossRef] [PubMed]

19. Kuznetsova, A.; Cullingham, C.; McKenzie, D.; Aiken, J.M. Soil humic acids degrade CWD prions and reduce infectivity. PLoS Pathog. 2018, 14, e1007414. [CrossRef]

20. Zabel, M.; Ortega, A. The ecology of prions. Microbiol. Mol. Biol. Rev. 2017, 81. [CrossRef]

21. Miller, M.W.; Williams, E.S.; Hobbs, N.T.; Wolfe, L.L. Environmental sources of prion transmission in mule deer. Emerg. Infect. Dis. 2004, 10, 1003-1006. [CrossRef] [PubMed]

22. Seidel, B.; Thomzig, A.; Buschmann, A.; Groschup, M.H.; Peters, R.; Beekes, M.; Terytze, K. Scrapie agent (Strain $263 \mathrm{~K}$ ) can transmit disease via the oral route after persistence in soil over years. PLoS ONE 2007, 2, e435. [CrossRef] [PubMed]

23. Maddison, B.C.; Owen, J.P.; Bishop, K.; Shaw, G.; Rees, H.C.; Gough, K.C. The interaction of ruminant PrPSc with soils is influenced by prion source and soil type. Environ. Sci. Technol. 2010, 44, 8503-8508. [CrossRef] [PubMed]

24. Saunders, S.E.; Yuan, Q.; Bartz, J.C.; Bartelt-Hunt, S. Effects of solution chemistry and aging time on prion protein adsorption and replication of soil-bound prions. PLOS ONE 2011, 6, e18752. [CrossRef]

25. Nagaoka, K.; Yoshioka, M.; Shimozaki, N.; Yamamura, T.; Murayama, Y.; Yokoyama, T.; Mohri, S. Sensitive detection of scrapie prion protein in soil. Biochem. Biophys. Res. Commun. 2010, 397, 626-630. [CrossRef]

26. Saunders, S.E.; Shikiya, R.A.; Langenfeld, K.; Bartelt-Hunt, S.L.; Bartz, J.C. Replication efficiency of soil-bound prions varies with soil type. J. Virol. 2011, 85, 5476-5482. [CrossRef]

(C) 2020 by the authors. Licensee MDPI, Basel, Switzerland. This article is an open access article distributed under the terms and conditions of the Creative Commons Attribution (CC BY) license (http://creativecommons.org/licenses/by/4.0/). 